\title{
Rectangular Potentials in a Semi-Harmonic Background: Spectrum, Resonances and Dwell Time ${ }^{\star}$
}

\author{
Nicolás FERNÁNDEZ-GARCÍA † and Oscar ROSAS-ORTIZ \\ ${ }^{\dagger}$ Instituto de Física, UNAM, AP 20-353, 01000 México D.F., Mexico \\ E-mail: nicolas@fisica.unam.mx \\ $\ddagger$ Physics Department, Cinvestav, A.P. 14-740, México DF 07000, Mexico \\ E-mail: orosas@fis.cinvestav.mx
}

Received December 01, 2010, in final form April 29, 2011; Published online May 05, 2011

doi:10.3842/SIGMA.2011.044

\begin{abstract}
We study the energy properties of a particle in one dimensional semi-harmonic rectangular wells and barriers. The integration of energies is obtained by solving a simple transcendental equation. Scattering states are shown to include cases in which the impinging particle is 'captured' by the semi-harmonic rectangular potentials. The 'time of capture' is connected with the dwell time of the scattered wave. Using the particle absorption method, it is shown that the dwell time $\tau_{D}^{a}$ coincides with the phase time $\tau_{W}$ of Eisenbud and Wigner, calculated as the energy derivative of the reflected wave phase shift. Analytical expressions are derived for the phase time $\tau_{W}$ of the semi-harmonic delta well and barrier potentials.
\end{abstract}

Key words: exactly solvable potentials; scattering process; resonances; Eisenbud-Wigner phase time; dwell time

2010 Mathematics Subject Classification: 35Q40; 35B34; 81U30; 81Q60

\section{Introduction}

One-dimensional models of quantum mechanics are useful in a number of applications in contemporary physics $[1,2,3,4,5,6,7,8,9,10,11,12,13,14,15,16,17,18]$. Often employed into the approximations which make tractable the more elaborated three-dimensional systems, they allow to get a deeper insight on the physics involved. Their simplicity has made them valuable as academic and research tools. For instance, the concept of effective mass, successfully applied in describing the formation of shallow energy levels due to impurities in crystals, leads to one-dimensional systems [1]. Localized lattice deformations can be modelled as a rectangular potential to study isolated transitions, observed in semiconductors, from a bound state within a quantum well to a bound state at an energy greater than the barrier height [2]. The analysis of resonances $[3,4,5,6,7,8]$ is transparent for rectangular potentials in either, the presence of a background interaction [9, 10], or in free space [11, 12, 13, 14, 15, 16, 17]. Simple models of point-like [18, 19], as well as regularized singular interactions [20], can be obtained as limit cases of rectangular potentials [21, 17, 22]. The one-dimensional models are also useful in the study of supersymmetric quantum mechanics [23, 24, 25, 26, 27]. Based on the Darboux transformations [28] (see also [23]), the supersymmetric (intertwining or factorization) formalism allows the construction of new exactly solvable potentials even if complex energies are involved $[29,30,31,32,33]$. This last property has been implemented to get one-dimensional complex potentials behaving as an optical device which both refracts and absorbs light waves [15, 33].

${ }^{\star}$ This paper is a contribution to the Proceedings of the Workshop "Supersymmetric Quantum Mechanics and Spectral Design" (July 18-30, 2010, Benasque, Spain). The full collection is available at http://www.emis.de/journals/SIGMA/SUSYQM2010.html 
Collisions, on the other hand, are modelled as interactions localized in time and space. This implies that the involved potential vanishes rapidly enough in space, so that incoming and outgoing asymptotic states can be represented by wave packets in free motion. The problem is usually reduced to the analysis of a one-dimensional effective potential. Main information is then obtained from the transmission and reflection amplitudes. Of particular interest, the resonance phenomenon is experimentally studied in atomic, nuclear and particle physics $[3,4,5,6]$. A resonance can be understood as an special result of the scattering process in which the incident wave is 'captured' by the scatterer for a while. A measurable time delay (relative to free motion) is then associated to the scattered wave [34, 35].

The concept of time delay corresponds to the time spent by an scattered particle in the scattering zone when compared to a free particle subject to the same initial conditions. According to Eisenbud and Wigner, this is associated to the energy derivative of the phase shift for binary collisions (see [36, 37]). The existence of a global time delay (as connected to sojourn times), and its identity with the Eisenbud-Wigner phase time, have been proved for local potentials in $\mathbb{R}^{3}$ [38]. The proof has been extended to an abstract formalism where time delay is defined in terms of the expectation values of non-negative normalized functions of compact support [39]. In general, the retardation of the scattered wave involves transient effects which are relevant in nuclear reactions [40, 41], and is also fundamental in the characterization of resonances [34, 35]. The time delay is also connected with the Levinson theorem [42], the density of states in mesoscopic conductors [43] and with the study of the photodetachment rate due to weak time-periodic electric fields [44]. In one-dimensional systems, the time delay has been studied for step-like potentials exhibiting a two-channel structure [45]. Quite recently, it has been shown that time delay can be obtained from the eigenvalues of a non-Hermitian Hamiltonian in two and three dimensions [46]. Extensive, clear reviews with complete bibliography can be found in [47, 48, 49].

The present work is addressed to the analysis of the energy properties of a particle subject to the action of a semi-harmonic rectangular potential. This last is either a rectangular well or barrier in a background integrated by a free-particle interaction to the right and an oscillatorlike interaction to the left of the rectangular potential. The model corresponds to a system (the rectangular potential) embedded in an environment (the semi-harmonic background), and the issue is the study of the modifications on the energy spectrum and resonances of the system which are induced by the environment. Thus, the semi-harmonic square potentials are viewed as open one-dimensional quantum systems [5]. The bound states and resonances of square potentials have been studied by using diverse approaches (see, e.g., $[14,15,16]$ and references quoted therein). In particular, if closer resonances imply narrower widths (i.e., in the case of isolated resonances), the transmission amplitude $T$ can be written as a superposition of Fock-BreitWigner distributions [15]. Then, the position $E$ and width $\Gamma$ of a resonance $\epsilon=E-\frac{i}{2} \Gamma$ are in correspondence with one of the bell-shaped peaks of $T$. This result is extended to the case of resonances which are not isolated by identifying the position of the peaks with the absolute value of $\epsilon$, rather than using $\operatorname{Re}(\epsilon)=E$ [16]. In the limit where the square potentials become a point-like interaction (i.e., a delta barrier or well), the transparency properties involved produce no resonances [18]. The situation is different for dipole-like interactions represented by derivatives of the delta distribution with respect to the position [17].

As open systems, rectangular potentials have been previously studied in a static field as the environment [9, 10]; the reports include the point-like limit [44]. Unlike the rectangular potentials in the free-particle background, these last open systems are not isotropic since the environment is represented by a potential diverging as $\pm x$ at $|x|=+\infty$. Thus, though the presence of resonances is ensured by adding such a background, the conventional definitions of dwell time and time delay are not automatically applicable in these cases. Indeed, most of the approaches on the matter consider a potential which is very localized in a finite region of space [47, 48, 49] (see also [38]). In contraposition, the open systems presented in this paper become zero when 
they are evaluated at positions to the right of the square potential. This condition represents an advantage over the square potentials in a constant field environment since reflection times can be now calculated. In this context, the mean time spent by the particle in the interaction zone is defined as the dwell time (or sojourn time) involved. However, it must be emphasized that the parabolic part of the environment makes not simple the calculation of transmission times. Thereby, there is not a clear definition of time delay in terms of dwell time differences in arbitrary open balls of the one-dimensional domain of these systems. Instead, the time delay can be defined as the difference between the reflection times above mentioned. Here, we shall show that the dwell time coincides with the phase time introduced by Eisenbud and Wigner.

The organization of the paper is as follows. In Section 2 we establish the general expressions concerning the semi-harmonic rectangular potentials. The condition for the trapping of particles is identified as the finding of zeros of the Jost function. A very simple transcendental equation is derived for the numerical integration of energies. In Section 3, the family of semi-harmonic rectangular wells of unit area is shown to converge to a semi-harmonic delta well in the sense of distribution theory. The corresponding transcendental equation is shown to admit a unique root associated to bound states, and the result is compared with the single bound energy of the delta well in a free particle background. The resonances obey a rule of distribution in the complex plane of the energies that resembles the distribution of the odd bound energies of a harmonic oscillator. Section 4 is devoted to the application of the previous results to the case of a semi-harmonic rectangular barrier. Interestingly, the limit case of a semi-harmonic delta barrier obeys a distribution of resonances which is in correspondence with the even energy eigenvalues of the harmonic oscillator. A brief discussion on the Darboux transformations of rectangular potentials in a semi-harmonic background is also given. In Section 5 we investigate the dwell time of the scattering process associated to the semi-harmonic rectangular potentials. This is defined as the time spent by the incident particle in the interaction zone. We use the particle absorption method $[50,51,52]$ to determine the characteristic time involved. Then we assume that the probability of finding the particle decays exponentially from the moment that it is 'captured' by the potential. The dwell time so calculated coincides with the phase time $\tau_{W}$ of Eisenbud and Wigner. For delta wells and barriers explicit analytical expressions of $\tau_{W}$ are given. In each case, the time delay $\tau_{W}$ has local maxima centered at the real part of the involved resonances. This last, combined with the results of Sections 3 and 4 for delta-like potentials, indicates that the semi-harmonic background induces the real part of the resonances to be distributed in correspondence with the energy eigenvalues of the harmonic oscillator. Some concluding remarks are given in Section 6. Finally, a short appendix includes some derivations in terms of the confluent hypergeometric functions which, although important, can be postponed to a later reading.

\section{Semi-harmonic rectangular well}

Consider a particle of energy $E$ which is under the influence of the one-dimensional potential

$$
V(x ; a)=\left\{\begin{aligned}
x^{2}, & x \leq-a \quad(\text { Region I), } \\
-V_{0}, & |x|<a \quad(\text { Region II }), \quad a \geq 0, \quad V_{0}>0 . \\
0, & a \leq x \quad(\text { Region III }),
\end{aligned}\right.
$$

Potential (2.1) is depicted in Fig. 1. The corresponding stationary dimensionless Schrödinger equation

$$
(H-E) \psi(x)=0, \quad H:=-\frac{d^{2}}{d x^{2}}+V(x, a)
$$




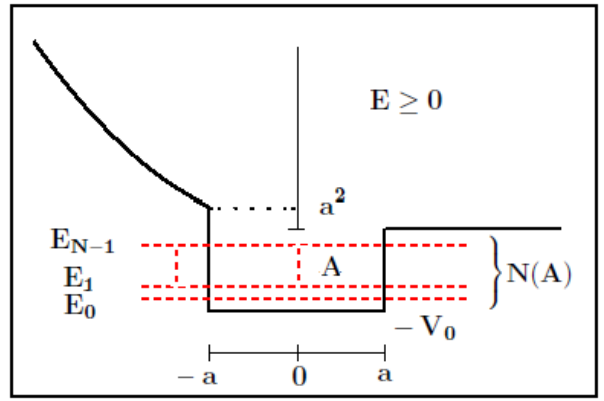

Figure 1. The rectangular well in a semi-harmonic background. The number $N$ of bound state energies $E_{0}, \ldots, E_{N-1}$, depends on the area $A=2 a V_{0}$ of the well (cf. Section 2.1.1). Some of the incoming scattering waves of energy $E \geq 0$ are 'captured' for a while in the region $(-\infty, a)$, as discussed in Section 5. If $V_{0}^{-1}=2 a$, the limit $a \rightarrow 0$ leads to a semi-harmonic delta well (see Section 3). Semiharmonic rectangular and delta barriers are obtained by the change $V_{0} \rightarrow-V_{0}$ (cf. Section 4).

is solved by the appropriate combination of the functions

$$
\begin{aligned}
& \psi_{\mathrm{I}}(x)=A_{1} e^{-x^{2} / 2}{ }_{1} F_{1}\left(\alpha, \frac{1}{2} ; x^{2}\right)+B_{1} e^{-x^{2} / 2} x_{1} F_{1}\left(\alpha+\frac{1}{2}, \frac{3}{2} ; x^{2}\right), \\
& \psi_{\mathrm{II}}(x)=A_{2} u(x)+B_{2} v(x):=A_{2} \sin q x+B_{2} \cos q x, \\
& \psi_{\mathrm{III}}(x)=A_{3} e^{i k x}+B_{3} e^{-i k x}
\end{aligned}
$$

where $k=\sqrt{E}$ and $q=\sqrt{V_{0}+k^{2}}$, the functions $e^{i k x}$ and $e^{-i k x}$ represent waves moving towards the right and the left respectively, and ${ }_{1} F_{1}(\alpha, \gamma ; z)$ stands for the confluent hypergeometric function (see Appendix) with $\alpha=\frac{1-k^{2}}{4}$.

To analyze the solutions of (2.2) as $x \rightarrow-\infty$, we use (A.3). A simple calculation gives

$$
\psi_{\mathrm{I}}(x) \approx \frac{\sqrt{\pi}}{\Gamma_{\mathrm{e}}(\alpha)} e^{\frac{x^{2}}{2}}\left(x e^{-i \pi}\right)^{2 \alpha-1}\left[A_{1}-\frac{B_{1}}{2} \frac{\Gamma_{\mathrm{e}}(\alpha)}{\Gamma_{\mathrm{e}}(\alpha+1 / 2)}\right], \quad \text { as } \quad x \rightarrow-\infty,
$$

with $\Gamma_{\mathrm{e}}(z)$ the Euler gamma function of $z \in \mathbb{C}$. Therefore, to construct solutions which are regular at the left edge of Dom $V(x, a)=(-\infty, \infty)$, the coefficient $B_{1}$ is constrained to satisfy

$$
B_{1}=2 A_{1} \frac{\Gamma_{\mathrm{e}}(\alpha+1 / 2)}{\Gamma_{\mathrm{e}}(\alpha)}
$$

As a consequence, the function $\psi_{\mathrm{I}}$ reads

$$
\psi_{\mathrm{I}}(x)=A_{1} e^{-x^{2} / 2}\left[{ }_{1} F_{1}\left(\alpha, \frac{1}{2} ; x^{2}\right)+2 x \frac{\Gamma_{\mathrm{e}}(\alpha+1 / 2)}{\Gamma_{\mathrm{e}}(\alpha)}{ }_{1} F_{1}\left(\alpha+\frac{1}{2}, \frac{3}{2} ; x^{2}\right)\right]:=A_{1} \varphi(x),
$$

and it becomes zero as $x \rightarrow-\infty$. If the parabolic part of the potential appears to the right, rather than to the left of the well, it occurs a phase difference in the asymptotic behavior of the solution (compare equations (A.2) and (A.3)). We decided to put the oscillator-like interaction to the left of the well to precisely emphasize the subtleties of the involved mathematics.

The condition of continuity at $x=-a$ leads to the coefficients

$$
\frac{A_{2}}{A_{1}}=\left.\frac{\varphi v\left(\beta_{v}-\beta_{\varphi}\right)}{q}\right|_{x=-a}:=\varphi(-a) C_{2}, \quad \frac{B_{2}}{A_{1}}=\left.\frac{\varphi u\left(-\beta_{u}+\beta_{\varphi}\right)}{q}\right|_{x=-a}:=\varphi(-a) D_{2},
$$

where $-\beta_{f}$ stands for the logarithmic derivative of the function $f(x)$. Hence we have

$$
\psi_{\mathrm{II}}(x)=A_{1} \varphi(-a)\left[C_{2} \sin q x+D_{2} \cos q x\right] .
$$


In turn, from the condition of continuity at $x=a$, one arrives at the expressions

$$
\frac{B_{3}}{A_{1}}=\varphi(-a) \frac{i}{2} \mathcal{F}(k, a), \quad \frac{A_{3}}{A_{1}}=\left(\frac{B_{3}}{A_{1}}\right)^{*} .
$$

Here $z^{*}$ is the complex conjugate of $z \in \mathbb{C}$, and the Jost function $\mathcal{F}(k, a)$ is defined as

$$
\mathcal{F}(k, a)=-\left\{\frac{e^{i k x}}{k}\left[u\left(\beta_{-}+\beta_{u}\right) C_{2}+v\left(\beta_{-}+\beta_{v}\right) D_{2}\right]\right\}_{x=a},
$$

with $-\beta_{-}$the logarithmic derivative of $e^{-i k x}$. Thus, for the solution in region III we have

$$
\psi_{\text {III }}(x)=A_{1} \varphi(-a) \frac{i}{2}\left[\mathcal{F}(k, a) e^{-i k x}-\mathcal{F}^{*}(k, a) e^{i k x}\right] .
$$

Scattering states. Consider now a particle of energy $E=k^{2}>0$, arriving from $+\infty$ towards the effective zone of the semi-harmonic well (2.1). The reflection amplitude

$$
s(k, a)=\frac{\mathcal{F}^{*}(k, a)}{\mathcal{F}(k, a)}
$$

is directly obtained from (2.6) and has modulus one. This can be written as $s(k, a)=e^{2 i \delta(k, a)}$. We see that the effect of the semi-harmonic rectangular well is to cause a phase shift (up to integer multiples of $\pi$ ) of the reflected wave by

$$
\delta(k, a)=-\arctan \left[\frac{\operatorname{Im} \mathcal{F}(k, a)}{\operatorname{Re} \mathcal{F}(k, a)}\right] .
$$

Then, for scattering states, the solution of (2.2) cancels as $x \rightarrow-\infty$, oscillates harmonically in region II (see equation (2.4)), and behaves as

$$
\psi_{\mathrm{III}}(x)=A_{1} \varphi(-a)|\mathcal{F}(k, a)| \sin (k x+\delta)
$$

in region III.

\subsection{Conditions for the trapping of particles}

The solutions of the Schrödinger equation (2.2) which correspond to either bound or resonance states are picked out from $(2.3),(2.4)$ and (2.6) such that only outgoing waves exist. In other words, to construct these solutions, it must be imposed the Siegert condition

$$
\lim _{x \rightarrow+\infty} \beta_{\psi}(x)=-i k, \quad k \in \mathbb{C} .
$$

This last expression is equivalent to make $\mathcal{F}(k, a)=0$ in (2.6). Then, the Jost function (2.5) is analytically continued so that the reflection amplitude (2.7) is a meromorphic function with poles on the zeros of $\mathcal{F}(k, a)$. For simplicity, hereafter $A_{1}=1 / \varphi(-a)$. Therefore, the wave functions of either bound states or resonances are written as

$$
\psi(x)=\left\{\begin{array}{ll}
\frac{\varphi(x)}{\varphi(-a)}, & x \leq-a, \\
C_{2} \sin q x+D_{2} \cos q x, & |x|<a, \\
-\frac{i}{2} \mathcal{F}^{*}(k, a) e^{i k x}, & a \leq x,
\end{array} \quad \text { such that } \mathcal{F}(k, a)=0 .\right.
$$

Given $a \neq 0$, the zeros of the Jost function $\mathcal{F}(k, a)$ are defined by the roots of the transcendental equation

$$
\beta_{\varphi}(-a)=-i k+\left[\frac{i k \beta_{\varphi}(-a)-q^{2}}{q}\right] \tan 2 q a .
$$

It is straightforward to verify that this last equation has no solutions on the real line. 
Table 1. The dimensionless single bound state energy of a unit area rectangular well in the free-particle $(\mathrm{FP})$ and semi-harmonic (SH) backgrounds for different values of $a$. In both cases, the numerical results include the delta well as a limit $(a \rightarrow 0)$.

\begin{tabular}{|c|c|c|}
\hline \hline$a$ & $\begin{array}{c}\text { rectangular well } \\
(\mathrm{FP})\end{array}$ & $\begin{array}{c}\text { rectangular well } \\
(\mathrm{SH})\end{array}$ \\
\hline 2.0 & -0.113438 & -0.045272 \\
1.5 & -0.130400 & -0.039482 \\
1.0 & -0.153960 & -0.033514 \\
0.5 & -0.189338 & -0.037435 \\
0.0 & -0.25 & -0.079710 \\
\hline
\end{tabular}

\subsubsection{Bound states}

Potential (2.1) includes a region of classical confinement in which some bound state energies could be present. In this context, if $k=i \kappa$ is a zero of the Jost function with $\kappa>0$, then $E=k^{2}=-\kappa^{2}$ is the energy eigenvalue of a bound state. Indeed, if $k$ is a solution of (2.11) on the positive imaginary axis of the complex $k$-plane, one has $\alpha=\frac{1-k^{2}}{4}=\frac{1+\kappa^{2}}{4}>0$, and $\beta_{\varphi} \in \mathbb{R}$. The number $N$ of these roots is determined by the area $A=2 a V_{0}$ of the well. Thereby, the bound states are represented by the square-integrable functions

$$
\begin{aligned}
& \psi_{n}(x)= \begin{cases}\frac{\varphi_{n}(x)}{\varphi_{n}(-a)}, & x \leq-a, \\
C_{2} \sin q_{n} x+D_{2} \cos q_{n} x, & |x|<a, \\
\left.\frac{u\left(\beta_{u}+\kappa_{n}\right) C_{2}+v\left(\beta_{v}+\kappa_{n}\right) D_{2}}{2 \kappa_{n}}\right|_{x=a} e^{-\kappa_{n}(x-a)}, & a \leq x,\end{cases} \\
& n=0,1, \ldots, N(A)-1 .
\end{aligned}
$$

For instance, if $V_{0}^{-1}=2 a$, potential (2.1) becomes a unit area rectangular well in a semi-harmonic background. This admits a unique bound state, just as it is reported on Table 1 for five different values of $a$ (see also Fig. 2). For comparison, the equivalent results for a free-particle background are also reported. In both cases, the single bound energy goes to a definite negative number as $a \rightarrow 0$. However, given $a \geq 0$, this energy is less negative in the semi-harmonic background than in the free-particle one. The same effect is observed for all the bound states belonging to a rectangular well of arbitrary area $A \neq 0$. Thus, in a semi-harmonic background, the bound states of the rectangular well are displaced towards the threshold. Notice that the oscillatorlike interaction to the left of a given (symmetric) well produces a left-right asymmetry in the involved wave functions (see, e.g., Fig. 2).

\subsubsection{Resonances}

Siegert functions are solutions of the Schrödinger equation (2.2), associated to complex eigenvalues $\epsilon=E-i \frac{\Gamma}{2}$, and fulfilling the purely outgoing condition (2.9). It is usual to consider $\epsilon$ as a compound of the resonance position $E$ and the inverse of the involved lifetime $\tau^{-1}=\Gamma$. We obtain these complex eigenvalues from the wave numbers $k$ in the fourth quadrant of the complex $k$-plane which are solutions of (2.11). Some of the first resonances associated to a semi-harmonic rectangular well of unit area are reported on Table 2 for different values of $a$. In contraposition to their equivalents in a free-particle background, where the symmetry of the rectangular well is inherited to the wave functions and resonances (see e.g. [14] and [15]), the Siegert functions of the rectangular well in a semi-harmonic background cancel at $x=-\infty$, obeying the left-right asymmetry generated by the oscillator-like interaction. This is illustrated in Fig. 3. 


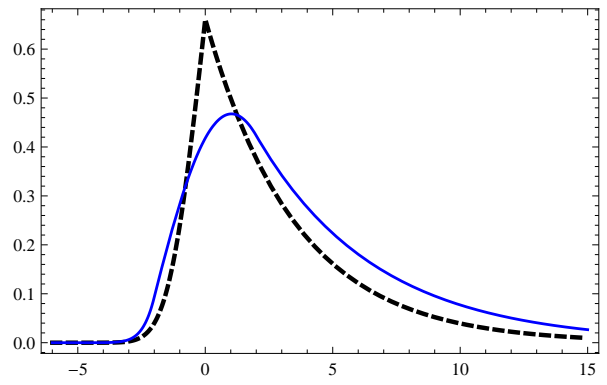

Figure 2. The wave function of the unique bound state belonging to the semi-harmonic rectangular well of unit area with $a=2$ (continuous, blue) and $a=0$ (dashed, black). The dimensionless energies are $E_{a=2}=-0.045272$ and $E_{\delta}=-0.079710$ respectively (see Table 1). Notice the left-right asymmetry produced by the parabolic part of potential (2.1).

Table 2. The first five resonances $\epsilon=E-i \frac{\Gamma}{2}$ belonging to the unit area rectangular well in a semiharmonic background for different values of $a$. The case $a=0$ (the semi-harmonic delta well) is such that $\operatorname{Re}(\epsilon)=4 m+3+\gamma_{m}$, with $\gamma_{m} \lesssim 1$ and $m=0,1, \ldots$ (see Fig. 5).

\begin{tabular}{|c|c|c|c|}
\hline \hline$a$ & resonances & $a$ & resonances \\
\hline \multirow{5}{*}{2} & $00.623117-i 0.599545$ & & $03.569260-i 1.487849$ \\
& $05.260402-i 2.255076$ & & $10.233701-i 2.499541$ \\
& $08.875649-i 2.603548$ & 0.5 & $13.960171-i 2.280915$ \\
& $11.217715-i 1.995880$ & & $17.904306-i 2.258438$ \\
& $17.359977-i 2.229026$ & & $21.878702-i 2.356575$ \\
\hline \multirow{5}{*}{1.5} & $01.009578-i 0.981433$ & & $03.792859-i 0.909297$ \\
& $03.852457-i 2.106492$ & & $07.852027-i 1.117703$ \\
& $07.574481-i 2.017753$ & $5 \times 10^{-4}$ & $11.880118-i 1.242053$ \\
& $09.826328-i 2.651538$ & & $15.897157-i 1.331170$ \\
& $13.723866-i 2.196828$ & & $19.908829-i 1.400707$ \\
\hline \multirow{5}{*}{1} & $01.838241-i 1.632446$ & & $03.792839-i 0.909196$ \\
& $05.675163-i 1.760804$ & & $07.852012-i 1.117599$ \\
& $08.749583-i 2.720863$ & 0 & $11.880106-i 1.241947$ \\
& $11.835116-i 2.294470$ & & $15.897146-i 1.331064$ \\
& $15.830298-i 2.343309$ & & $19.908819-i 1.400600$ \\
\hline \hline
\end{tabular}

\section{The semi-harmonic delta well}

A particular case of the previous results which deserves special attention is obtained in the limit $a \rightarrow 0$. It is well known that the family of rectangular wells of unit area converges to the delta well in the sense of distribution theory (see e.g. [21] and [17]). The transparency properties of the point interactions are such that no resonances can be associated to $\pm \Omega \delta(x)$, with $\Omega$ an opacity parameter [18]. The situation changes if the free-particle background is replaced by a less trivial scenario. Namely, the presence of resonances is ensured for the point interactions either by putting a constant potential as the background [9, 44] (see also [10]), or by constraining the potential domain of $\delta\left(x-x_{0}\right)$ to be $[0,+\infty)$ rather than the straight-line $\mathbb{R}$ [18]. In contradistinction, point dipole interactions represented by the derivative of the delta distribution $\pm \delta^{\prime}(x)$ admit resonances in a natural form [17]. We are going to analyze the bound states and resonances associated to a delta well in the semi-harmonic background introduced in the previous section. 

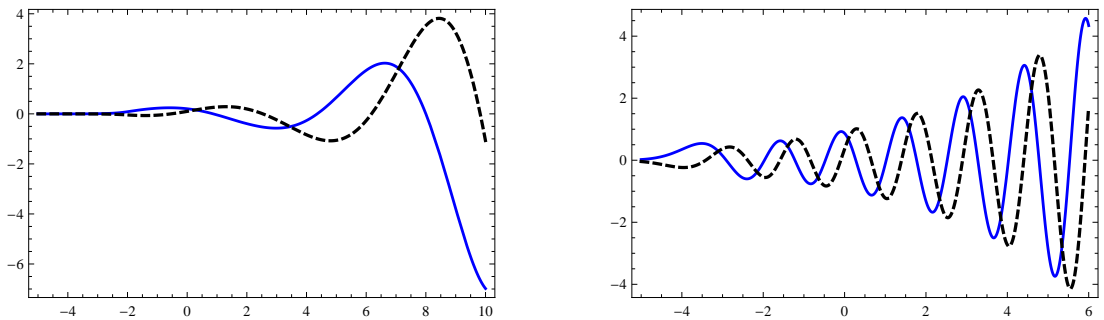

Figure 3. The real (continuous, blue) and imaginary (dashed, black) parts of the Siegert function belonging to the first (left) and fifth (right) resonances reported on Table 2 for $a=2$. In all cases, the left-right asymmetry is due to the parabolic part of potential (2.1).

Potential (2.1) represents a family of functions of compact support (the unit area rectangular wells, parameterized by $a$ ) in a semi-harmonic background. If $a \rightarrow 0$, we have

$$
V_{\delta}(x):=\lim _{a \rightarrow 0} V(x ; a)=V_{s}(x)-\delta(x),
$$

with

$$
V_{s}(x)= \begin{cases}x^{2}, & x<0 \\ 0, & x>0\end{cases}
$$

(details of the limit procedure for this kind of potentials can be consulted in [21, 22]). Applying the same limit to the transcendental equation (2.11), one gets the expression

$$
2 \Gamma_{\mathrm{e}}\left(\frac{3-k^{2}}{4}\right)=(1+i k) \Gamma_{\mathrm{e}}\left(\frac{1-k^{2}}{4}\right) .
$$

Equation (3.1) admits an isolated root on the positive imaginary axis of the complex $k$-plane (see Fig. 4). We have $k_{\delta}=i 0.2823302$. The corresponding energy $E_{\delta}=k_{\delta}^{2}=-0.0797104$, is consistently recovered as the limit $a \rightarrow 0$ of the values reported on Table 1 . Interestingly, this bound energy is closer to zero than the bound energy of the delta well in a free-particle background $E=-0.25$. That is, the semi-harmonic background causes the displacement of the single bound energy towards the threshold, just as it has been remarked above. The involved wave function

$$
\psi_{\delta}(x)= \begin{cases}\varphi(x), & x \leq 0 \\ e^{-\kappa x}, & 0<x\end{cases}
$$

is depicted in Fig. 2. Observe that the parabolic part of the potential produces a decreasing of $\varphi(x)$ which is faster than the decreasing of its counterpart $e^{-\kappa x}$ in the free particle zone.

In the present case, the resonances are determined by the roots of (3.1) in the fourth quadrant of the complex $k$-plane. Up to a constant $\gamma_{m}$, the position $E_{m}$ of each of these 'complex energies' is clearly connected to the distribution of the odd energy eigenvalues of the harmonic oscillator: $\operatorname{Re}(\epsilon)=4 m+3+\gamma_{m}$, with $m=0,1,2, \ldots$ For the results reported on Table 2, the parameter $\gamma_{m}$ approaches 1 from below as $m$ increases (see Fig. 5). As a conclusion, different than the conventional delta well, a delta well in a semi-harmonic background admits resonances. More details will be given in Section 5 .

\section{Semi-harmonic barriers}

The case of a particle in a semi-harmonic rectangular barrier can be analyzed as a consequence of the previous results. The change $V_{0} \rightarrow-V_{0}$ in $(2.1),(2.2)$ produces $q \rightarrow i \sqrt{\left|k^{2}-V_{0}\right|}$ for 


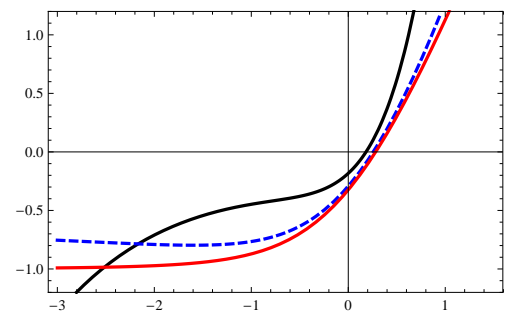

Figure 4. The isolated zero of the Jost function which corresponds to the unique bound state of a semiharmonic rectangular well of unit area for $a=1$ (continuous, black), $a=0.1$ (dashed, blue) and $a=0$ (continuous, red). In each case, the curve has been depicted as a function of $k=i \kappa$, with $\kappa \in \mathbb{R}$.
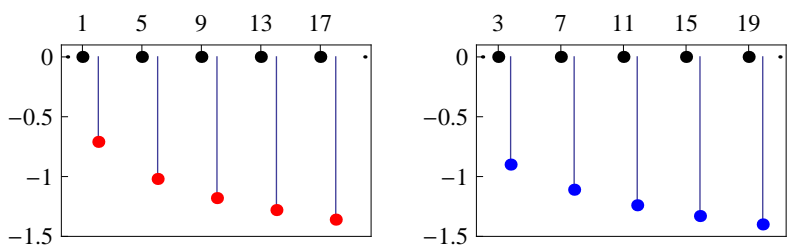

Figure 5. First five resonances $\epsilon=E-i \frac{\Gamma}{2}$ of a delta barrier (left, red disks) and a delta well (right, blue disks) in a semi-harmonic background. Black disks on the real line represent the even and odd energies of the harmonic oscillator respectively. Vertical lines are included as a reference. The numerical values of $\epsilon$ can be consulted on Tables 3 and 2 respectively.

$k^{2}<V_{0}$, and $q \rightarrow \sqrt{k^{2}-V_{0}}$ for $k^{2}>V_{0}$. The straightforward calculation shows that there is no bound states for this system. Some of the corresponding resonances, in turn, are reported on Table 3 (see also Fig. 6). In this case, the complex energies $\epsilon$ are below and close to the positive real axis such that, in the very limit $a \rightarrow 0, \operatorname{Re}(\epsilon)$ is in one of the positions $4 m+1+\lambda_{m}$, with $\lambda_{m}$ a constant and $m=0,1,2, \ldots$ For the results on Table $3, \lambda_{m}$ approaches 1 from above as $m$ increases (see Fig. 5). Then, the resonances of a delta barrier in a semi-harmonic background are distributed in correspondence with the even energy eigenvalues of the harmonic oscillator. See more details in Section 5.

\subsection{Darboux transformations}

The Siegert condition (2.9) is appropriate to construct the complex supersymmetric partners of the semi-harmonic potentials analyzed in the previous sections. Consider the Darboux transformation

$$
\widetilde{V}(x ; a)=V(x ; a)+2 \beta^{\prime}(x),
$$

with $V(x ; a)$ either a semi-harmonic well or barrier, and $\beta(x)$ the logarithmic derivative of any of the corresponding Siegert functions $\psi_{\epsilon}$. Condition (2.9) indicates that the new potential $\widetilde{V}(x ; a)$ behaves as $V(x ; a)$ in the limit $x \rightarrow+\infty$. On the other hand, the straightforward calculation shows that $\beta(x) \rightarrow 0$, as $x \rightarrow-\infty$. Therefore, $\widetilde{V}(x ; a)$ is a complex potential behaving as $V(x ; a)$ at the edges of Dom $V(x ; a)=(-\infty,+\infty)$. The supersymmetric formalism of quantum mechanics $[28,23,24,25,26,27]$ ensures that the energy spectrum of $\widetilde{V}(x ; a)$ is the same as that of $V(x, a)$ if the superpotential is defined as $\beta(x)=-\frac{d}{d x} \ln \psi_{\epsilon}(x)$ (cf. [15, 30, 31, 32, 33]). In Fig. 7 we show the behavior of the Darboux-deformed semi-harmonic rectangular barrier, constructed from (4.1) with the Siegert state belonging to the fifth resonance of Table 3. The presence of maxima and minima, in both the real and imaginary parts of the potential, makes $\widetilde{V}(x ; a)$ to behave as an optical device which both refracts and absorbs light waves [23, 15]. A similar result 
Table 3. The first five resonances $\epsilon=E-i \frac{\Gamma}{2}$ belonging to the unit area rectangular barrier in a semi-harmonic background for different values of $a$. The case $a=0$ (the semi-harmonic delta barrier) is such that $\operatorname{Re}(\epsilon)=4 m+1+\lambda_{m}$, with $\lambda_{m} \gtrsim 1$ and $m=0,1,2, \ldots$ (see Fig. 5 ).

\begin{tabular}{|c|c|c|c|}
\hline \hline$a$ & resonances & $a$ & resonances \\
\hline \multirow{5}{*}{2} & $00.595222-i 0.312336$ & & $01.957470-i 0.802720$ \\
& $04.211813-i 1.853317$ & & $05.720407-i 1.561124$ \\
& $07.387244-i 2.485184$ & 0.5 & $09.297545-i 2.218137$ \\
& $10.572524-i 1.985917$ & & $12.626496-i 2.505935$ \\
& $12.865432-i 2.874092$ & & $16.213699-i 2.433756$ \\
\hline \multirow{5}{*}{1.5} & $00.846017-i 0.497624$ & & $02.076264-i 0.718026$ \\
& $06.188284-i 2.213815$ & & $06.065577-i 1.025854$ \\
& $08.833155-i 2.011628$ & $5 \times 10^{-4}$ & $10.058023-i 1.181640$ \\
& $12.476467-i 2.723854$ & & $14.052696-i 1.286283$ \\
& $14.938732-i 2.618887$ & & $18.048705-i 1.360061$ \\
\hline \multirow{5}{*}{1} & $01.313699-i 0.776043$ & & $02.076211-i 0.718123$ \\
& $04.317689-i 1.833635$ & & $06.065549-i 1.025956$ \\
& $07.338936-i 2.031873$ & 0 & $10.058003-i 1.181793$ \\
& $10.901777-i 2.284989$ & & $14.052679-i 1.286389$ \\
& $14.229903-i 2.730174$ & & $18.048690-i 1.365109$ \\
\hline \hline
\end{tabular}
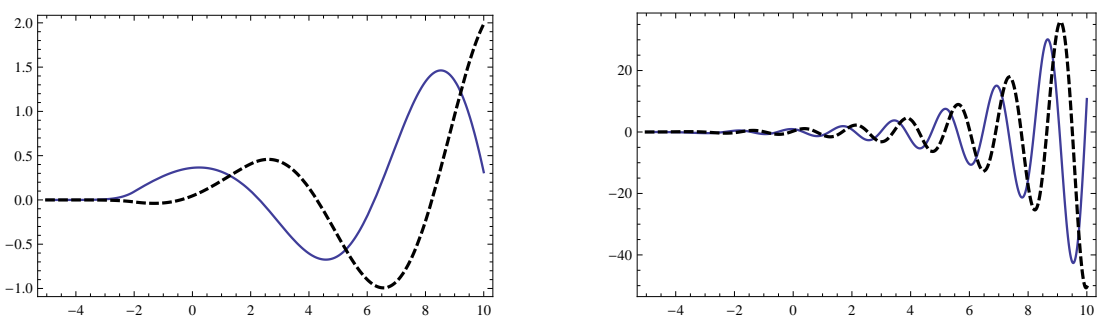

Figure 6. The real (continuous, blue) and imaginary (dashed, black) parts of the Siegert function belonging to the first (left) and fifth (right) resonances reported on Table 3 for $a=2$. Remark that the left-right asymmetry of all these functions is generated by the parabolic part of potential (2.1).

is obtained for the Darboux-deformations of the semi-harmonic wells. The procedure can be repeated at will, giving rise to more elaborate complex deformations of the initial potentials. Particularly interesting, the double transformation obtained by using a complex eigenvalue $\epsilon$ in a first step, and its complex conjugate $\epsilon^{*}$ in the second step, produces deformations which are real functions (see, e.g. [31, 15, 7]). In such a case, the semi-harmonic barriers will exhibit 'hair' as a characteristic of the deformation $[15,7]$.

\section{Dwell time}

As yet the bound states and resonances have been analyzed as different manifestations of the same sort of mathematical solution. Both of them are defined in terms of the zeros of the Jost function (2.5) and are represented by a Siegert state of the form (2.10). Concerning scattering states, let $E>0$ be the energy of a particle which impinges on a scatterer from the right, according to the rule (2.1). Here $V_{0}$ can be either positive or negative. Exterior to the interaction zone $(-\infty, a)$, the stationary scattering wave function is $\psi_{\mathrm{III}} \propto e^{-i k x}-s(k, a) e^{i k x}$ (see equations (2.6) and (2.7)). Since there is neither sources nor sinks we have total probability conservation $|s(k, a)|^{2}=1$. We want to get some insights on the time spent by the incident 

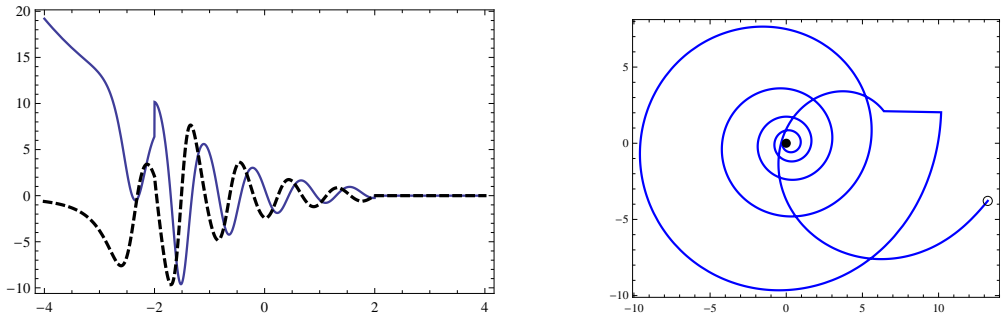

Figure 7. Left. The real (continuous, blue) and imaginary (dashed, black) parts of the supersymmetric partner $\widetilde{V}(x ; a)$ of a semi-harmonic rectangular barrier. This has been constructed with the fifth resonance for $a=2$. Right. The Argand diagram of $\widetilde{V}(x ; 2)$. The circle $\circ$ and disk $\bullet$ serve as reference and correspond to $\widetilde{V}(-3 ; 2)$ and $\widetilde{V}(3 ; 2)$ respectively.

particle in the interaction zone. To analyze the 'capturing' of the particle by the semi-harmonic rectangular potential, we assume $|s(k, a)|^{2}=e^{-\tau_{D}^{a} / \tau_{f}}$, with $\tau_{D}^{a}$ the mean time spent by the particle in the interaction zone $(-\infty, a)$, and $\tau_{f}$ a characteristic time constant to be determined. Using the absorption probabilities method [50, 51, 52], a small imaginary part $\Delta V_{I}$ is added to the potential under consideration. The absorption dimensionless Schrödinger equation to be solved is $\left(H+i \Delta V_{I}\right) \psi_{a}=i \frac{\partial \psi_{a}}{\partial t}$. Given a solution $\psi_{0}$ of the unperturbed equation (for which $\Delta V_{I}=0$ ), we use the ansatz $\psi_{a}=\psi_{0} g$ to get $\psi_{a}=\psi_{0} e^{\Delta V_{I} t}$ (an arbitrary integration constant has been omitted for simplicity). Therefore, the probability density of finding the particle is $\left|\psi_{0}\right|^{2} e^{2 \Delta V_{I} t}$. The time $\tau_{f}$ required for the probability density to decrease a factor $e^{-1}$ of its initial value $\left|\psi_{0}\right|^{2}$ is $\tau_{f}=-1 /\left(2 \Delta V_{I}\right)$, as this is introduced in [51]. The dwell time (or sojourn time) $\tau_{D}^{a}$ in the region $(-\infty, a)$ can be now obtained from $|s(k, a)|^{2}=e^{2 \tau_{D}^{a} \Delta V_{I}}$. Indeed, in the limit of small $\Delta V_{I}$, a simple calculation gives

$$
\tau_{D}^{a}=\frac{1}{2} \lim _{\Delta V_{I} \rightarrow 0} \frac{\partial}{\partial \Delta V_{I}}|s(k, a)|^{2} .
$$

Now, consider a solution of the absorption eigenvalue equation $\left(H+i \Delta V_{I}-\epsilon\right) \psi_{\epsilon}=0$, with $\epsilon \in \mathbb{C}$. The derivative with respect to $\Delta V_{I}$ of this last equation, after evaluating it for $\Delta V_{I}=0$, is reduced to

$$
i \psi_{\epsilon}+\left.(H-\epsilon) \frac{\partial \psi_{\epsilon}}{\partial \Delta V_{I}}\right|_{\Delta V_{I}=0}=0 .
$$

The derivative of the absorption eigenvalue equation with respect to $\epsilon_{I}$, the imaginary part of $\epsilon$, after evaluating it for $\Delta V_{I}=0$, produces

$$
-i \psi_{\epsilon}+(H-\epsilon) \frac{\partial \psi_{\epsilon}}{\partial \epsilon_{I}}=0
$$

Adding these last two results yields

$$
\lim _{\Delta V_{I} \rightarrow 0} \frac{\partial}{\partial \Delta V_{I}}=-\frac{\partial}{\partial \epsilon_{I}} \text {. }
$$

In this way, equation (5.1) can be rewritten as follows

$$
\tau_{D}^{a}=-\frac{1}{2} \frac{\partial}{\partial \epsilon_{I}}|s(k, a)|^{2} .
$$

Since $k^{2}=\epsilon$, the reflection amplitude $s(k, a)$ must be a complex analytic function of the complex eigenvalue $\epsilon=E+i \epsilon_{I}$. The Cauchy-Riemann condition reads as

$$
\frac{\partial s_{R}}{\partial E}=\frac{\partial s_{I}}{\partial \epsilon_{I}} \quad \text { and } \quad-\frac{\partial s_{R}}{\partial \epsilon_{I}}=\frac{\partial s_{I}}{\partial E} .
$$


The introduction of these last expressions into (5.2) produces

$$
\tau_{D}^{a}=2 \frac{\partial \delta}{\partial E}=\tau_{W}
$$

where we have used $s(k, a)=e^{2 i \delta(k, a)}$. From the above expression, the dwell time of the scattering particles which are 'trapped' in the semi-harmonic rectangular potentials is in direct connection with the slope of the reflected wave phase shift $\delta(E, a)$. Thereby, the dwell time (5.3) coincides with the definition of phase time $\tau_{W}$, introduced by Eisenbud and Wigner as the time delay in binary collisions [36]. In particular, the formation of a resonance $\epsilon=E_{r}-i \frac{\Gamma}{2}$ introduces a positive time delay between the arrival and departure of the scattering particle from the region $(-\infty, a)$. This last means that particles impinging the semi-harmonic rectangular potentials with energy $E=E_{r}$, will spend times in $(-\infty, a)$ which are larger than the times spent by the scattering particles of energy $E=E_{r}+\varepsilon$, with $\varepsilon \neq 0$ a real number small enough. A rapid increasing of the phase shift is then expected in the vicinity of the resonance position $E_{r}$. Hence, the phase shift (2.8) encodes enough information to identify true resonances by associating the peaks of (5.3) with the real part of the complex eigenvalues $\epsilon$ (see, e.g. [34]). Of special interest, the time delay of point interactions in a semi-harmonic background can be calculated as the limit $a \rightarrow 0$ of (5.3). One obtains

$$
\tau_{W}^{( \pm)}(E)=-2\left(\frac{1}{1+W_{ \pm}^{2}(E)}\right) \frac{d W_{ \pm}(E)}{d E}
$$

where the sign $+(-)$ stands for the semi-harmonic delta barrier (well) and

$$
W_{ \pm}(E)=\frac{\operatorname{Im}\left(\mathcal{F}_{ \pm}\right)}{\operatorname{Re}\left(\mathcal{F}_{ \pm}\right)}=\frac{-\Gamma_{\mathrm{e}}\left(\frac{1-E}{4}\right) \sqrt{E}}{ \pm \Gamma_{\mathrm{e}}\left(\frac{1-E}{4}\right)+2 \Gamma_{\mathrm{e}}\left(\frac{3-E}{4}\right)}, \quad \mathcal{F}_{ \pm}:=\mathcal{F}_{ \pm}(x, a \rightarrow 0)
$$

An straightforward calculation shows that $\tau_{W}^{( \pm)} \rightarrow \frac{\pi}{2}$, as $E \rightarrow+\infty$. From Fig. 8, we notice that $\tau_{W}^{(-)}\left(\tau_{W}^{(+)}\right)$exhibits a series of Fock-Breit-Wigner peaks W1, W2, . (B1, B2, ..), centered each one at the real part of the first, second, etc, energy resonances reported on Table 2 (Table 3). Thus, the zeros of the Jost function (2.5) are interrelated with the maxima of the time delay (5.4), as expected for true resonances [34]. A conclusion which makes self-consistent our approach since the same results are obtained in two different forms. From Fig. 8, we also notice that all the 'captured' particles spend a time $\tau_{W} \geq 1$ in $(-\infty, a)$, except for a region of small positive energies in the rectangular well case. The closer to the resonance position $\operatorname{Re}(\epsilon)$ is the incoming energy $E$, the larger is the time spent by the particle in the interaction zone.

In general, after entering the region $(-\infty, a)$ of an arbitrary semi-harmonic rectangular potential, the particle may be reflected back and forth many times before it manages to get back out (see discussions on the matter in [35]). These reflections may take place either in the zone of the rectangular potential $(-a, a)$, the parabolic part of the potential $(-\infty,-a)$, or in a combination of both. Our calculations consider the complete scattering process so that it is not possible to distinguish among all possible internal reflections and self-interference during the approach to the region $(-\infty, a)$. For an arbitrary isotropic potential $V_{0}$ (i.e., $V_{0}(x)$ becomes zero as $|x| \rightarrow+\infty)$, the dwell time $\tau_{D}$ must obey the identity $\tau_{D}=\tau_{R}|R|^{2}+\tau_{T}|T|^{2}$, with $R(T)$ the reflection (transmission) amplitude, and $\tau_{R}\left(\tau_{T}\right)$ the average time spent in $V_{0}$ by reflected (transmitted) particles. For instance, if $V_{0}$ is a rectangular barrier in the free-particle background, one has $\tau_{R}=\tau_{T}=\tau_{D}$, so that all particles spend, on average, the same amount of time in $V_{0}$, no matter the size of the barrier $[53,47,50]$. Our expression (5.2) is then associated to the reflection time, the mean time if particles are finally reflected, in the absorption model [51] (see also [53]). The comparison of $\tau_{D}^{a}$, as this has been defined in (5.3), with $\tau_{R}$ for the rectangular 


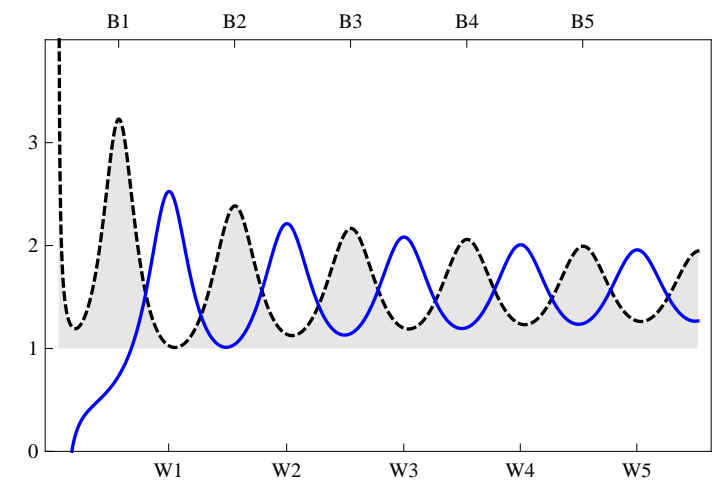

Figure 8. The Eisenbud-Wigner time delay $\tau_{W}$ for the scattering process of a semi-harmonic delta barrier (dashed, black) and well (continuous, blue). The peaks $\mathrm{W} n(\mathrm{~B} n)$ localize the real part of the resonances reported on Table 2 (Table 3 ).

barrier could give us additional information of the time delay induced by the semi-harmonic background.

Roughly speaking, time delay of the semi-harmonic rectangular potentials should correspond to the excess time that the scattering particle spends in $(-\infty, a)$, when compared to a free particle under the same initial conditions. Since our model considers the semi-harmonic background as the environment into which the square potential is embedded, this last can be taken as an open quantum system [5]. In this respect, the above indicated comparison of $\tau_{D}^{a}$ and $\tau_{R}$ makes sense in order to calculate time delays as the difference of reflection times. Some insights would be also obtained concerning transmission times. Given an open interval $\omega=\left(x_{1}, x_{2}\right)$ of sufficiently large size $x_{2}-x_{1}$ in $(-\infty, a)$, and a time window $\theta=\left(t_{1}, t_{2}\right)$ with $t_{2}>t_{1}$, one could introduce the corresponding dwell time as

$$
\Theta(\omega, \theta)=\int_{\theta} d t \int_{\omega} d x\left|e^{-i H t} \psi(x)\right|^{2}
$$

with $H$ as this has been given in (2.2), and $\psi$ an scattering state of energy $E$. In a similar form, the expression

$$
\Theta_{0}(\omega, \theta)=\int_{\theta} d t \int_{\omega} d x\left|e^{-i H_{0} t} \psi(x)\right|^{2}
$$

with $H_{0}$ the Hamiltonian of the square potential in a free-particle background, could be taken as the dwell time of the 'free-particle' system. The time delay in the space-temporal window $(\omega, \theta)$ should correspond to the difference $\Theta-\Theta_{0}$, as usual. In such a definition the quantities $\Theta$ and $\Theta_{0}$ are assumed to be well defined and finite for arbitrary $\omega \in(-\infty, a)$. In principle, it seems to be the case since all the involved scattering wave functions cancel at $x=-\infty$ (this is because the parabolic part of the environment). In order to arrive at a quantity which is independent of $\omega$, one could take the limits $x_{1} \rightarrow-\infty$ and $x_{2} \rightarrow a$. However, the anisotropy of the semi-harmonic rectangular potentials makes this last step not evident a priori. That is, some caution is required to apply the difference of sojourn times as the definition of time delay in the semi-harmonic rectangular potentials. Mainly, as connected with transmission dwell times in the interaction zone $(-\infty, a)$ since the potential diverges as $x^{2}$ at $x=-\infty$. Thus, in this last case, there is not a clear connection between sojourn times and phase times because almost all the approaches on the matter require potentials localized in a finite zone of the real line. The problem deserves a detailed analysis and the results will be reported elsewhere. 


\section{Concluding remarks}

In this paper, we studied the energy properties of a particle in the presence of a semi-harmonic rectangular potential. The latter is a one-dimensional rectangular potential in a background composed by an harmonic interaction to the left and a free particle interaction to the right of the rectangular potential. The advantage of this model lies in its simplicity to get analytic expressions for the wave functions, scattering states and resonances. Indeed, the problem is faced by considering bound states and resonances as different manifestations of the same sort of mathematical solution. Both of them are defined in terms of the zeros of the Jost function and are represented by a Siegert state. The numerical integration of the energies is done by solving a simple transcendental equation. In particular, it is found that, in the presence of a semi-harmonic background, the conventional bound energies of the rectangular well are displaced towards the threshold. Such behavior is preserved in the limit where the well becomes a delta. In this limit, there is a single bound state of energy $E_{\delta}=-0.079710$, which is less negative than its counterpart in a free-particle background, the value of which is $E=-0.25$. The resonances appearing for the semi-harmonic delta well exhibit a very peculiar behavior. They are distributed below and close to the positive real axis of the complex plane in such a way that their real part mimics the odd energy eigenvalues distribution of a harmonic oscillator. Namely, they are located according to the rule $4 m+3+\gamma_{m}$, with $\gamma_{m} \lesssim 1$ and $m=0,1,2, \ldots$. A similar situation occurs for the resonances of a semi-harmonic delta barrier, with the distribution ruled by $4 m+1+\lambda_{n}$ with

$\lambda_{m} \gtrsim 1$. Thus, in this case, the semi-harmonic background induces the real part of the resonances to be distributed in correspondence to the energy eigenvalues of the harmonic oscillator. The Siegert functions were successfully applied to construct complex Darboux-deformations of the semi-harmonic rectangular potentials. These new potentials behave as optical devices which both refract and absorb light waves.

Concerning the times involved in the scattering process, we assumed the time exponential decay rule $|s(k, a)|^{2}=\exp \left(-\tau_{D}^{a} / \tau_{f}\right)$, with $s(k, a)$ the reflection amplitude, $\tau_{f}$ a constant to be determined and $\tau_{D}^{a}$ the mean time spent by an scattering particle coming from $+\infty$ towards the interaction zone $(-\infty, a)$. The application of the absorption probability method $[50,51,52]$ allowed us to show that $\tau_{D}^{a}$ coincides with the phase-time of Eisenbud and Wigner $\tau_{W}$, calculated as the derivative of the reflected wave phase shift $\delta(E, a)$ with respect to the energy $E$. Becuase the global properties of the potentials we have discussed on, the dwell time so calculated corresponds to a reflection time (the mean time if particles are finally reflected). In the limit where the rectangular potentials become point-like interactions, we derived an explicit analytical expression for $\tau_{W}$, and showed that its local maxima are in correspondence with the resonances obtained in the previous sections. Finally, the semi-harmonic rectangular potentials can be viewed as the open quantum systems integrated by a rectangular potential (the system itself) and an environment (the semi-harmonic background). In this picture, the system is an isotropic potential for which the time spent in any finite region of space, averaged over all incoming particles, is well defined and corresponds to the dwell time $\tau_{D}$ involved. Therefore, the semi-harmonic rectangular potentials can be seen as the 'system' affected by a semi-harmonic interaction. Some insights on time delay can be obtained by calculating differences between the dwell time of these two systems in a given space-temporal window. This approach, however, is not directly applicable in the present case because the anisotropy of the environment. Work in this direction is in progress.

\section{A The confluent hypergeometric operator}

The kernel $\mathcal{K}_{(a, c)}$ of the confluent hypergeometric operator

$$
L_{(a, c)}:=z \frac{d^{2}}{d z^{2}}+(c-z) \frac{d}{d z}-a, \quad a, c \in \mathbb{R}, \quad z \in \mathbb{C}
$$




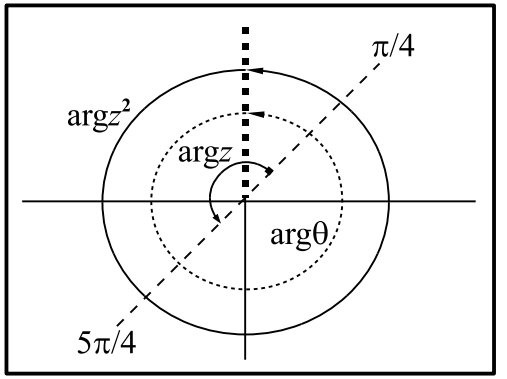

Figure 9. The two first sheets of the Riemann surface defined by the factor $e^{-a \pi i}$ in equation (A.1), the cut is at $\frac{\pi}{2}$. If $\frac{\pi}{4}<\arg z<\frac{5 \pi}{4}$, then $z^{2}$ and $\theta=e^{-2 \pi i} z^{2}$ are in the second and first sheets respectively.

is integrated by the solutions of the Kummer equation $L_{(a, c)} f(a, c ; z)=0$. That is, if $f(a, c ; z)$ is a confluent hypergeometric function then $f(a, c ; z) \in \mathcal{K}_{(a, c)}[54,55]$. The asymptotic expansion of $f(a, c ; z)$ for large values of $|z|$ is given by

$$
f(a, c ; z)=\frac{\Gamma(c)}{\Gamma(c-a)} e^{ \pm a \pi i} z^{-a}\left[1+P_{-}(a)\right]+\frac{\Gamma(c)}{\Gamma(a)} e^{z} z^{a-c}\left[1+P_{+}(c-a)\right]
$$

where the positive sign in the factor $e^{ \pm a \pi i}$ is taken when $-\frac{\pi}{2}<\arg z<\frac{3 \pi}{2}$, while the negative sign is taken when $-\frac{3 \pi}{2}<\arg z<\frac{\pi}{2}[56]$ and

$$
P_{ \pm}(\gamma)=\sum_{n=1}^{\infty}( \pm)^{n} \frac{(\gamma)_{n}(1-c+\gamma)_{n}}{n ! z^{n}}, \quad(\gamma)_{n}=\gamma(\gamma+1)(\gamma+2) \cdots(\gamma+n-1), \quad(\gamma)_{0}=1
$$

The above expressions can be utilized to deduce the asymptotic expansion of $f(a, c ; z)$ for specific ranges of $\arg z$. For instance, if $-\frac{\pi}{2}<\arg z<\frac{\pi}{2}$ we get

$$
f(a, c ; z) \rightarrow \frac{\Gamma(c)}{\Gamma(a)} e^{z} z^{a-c}\left[1+P_{+}(c-a)\right], \quad \operatorname{Re}(z)>0
$$

and

$$
f\left(a, c ; z^{2}\right) \approx \frac{\Gamma(c)}{\Gamma(a)} e^{z^{2}} z^{2(a-c)} \quad \text { if } \quad z \rightarrow+\infty
$$

To find the behavior of $f\left(a, c ; z^{2}\right)$ for $z \rightarrow-\infty$ we consider a range of $\arg z$ which includes the negative real values of $z$. For this, let us assume $\frac{\pi}{4}<\arg z<\frac{5 \pi}{4}$ (see Fig. 9). Then $\frac{\pi}{2}<\arg z^{2}<\frac{5 \pi}{2}$, and $z^{2}$ is in the second sheet of the Riemann surface defined by the factor $e^{-a \pi i}$ in (A.1). Since $\theta=e^{-2 \pi i} z^{2}$ is such that $-\frac{3 \pi}{2}<\arg \theta<\frac{\pi}{2}$, from (A.1) we arrive at

$$
f(a, c ; \theta)=\frac{\Gamma(c)}{\Gamma(c-a)} e^{a \pi i} z^{-2 a}\left[1+P_{-}(a)\right]+\frac{\Gamma(c)}{\Gamma(a)} e^{z^{2}} z^{2(a-c)} e^{-2 \pi i(a-c)}\left[1+P_{+}(c-a)\right] .
$$

Therefore, if $\arg z=\pi$ one finally gets

$$
f\left(a, c ; z^{2}\right) \approx \frac{\Gamma(c)}{\Gamma(a)} e^{z^{2}} z^{2(a-c)} e^{-2 \pi i(a-c)} \quad \text { if } \quad z \rightarrow-\infty .
$$

\section{Acknowledgements}

The support of CONACyT and DGAPA (UNAM) is acknowledged. 


\section{References}

[1] Bastard G., Theoretical investigations of super-lattice band-structure in the evelope-function approximation, Phys. Rev. B 25 (1982), 7584-7597.

[2] Capasso F., Sirtori C., Faist J., Sivco D.L., Chu S.-N.G., Cho A.Y., Observation of an electronic bound state above a potential well, Nature 358 (1992), 565-567.

[3] Brändas E., Elander N. (Editors), Resonances. The unifying route towards the formulation of dynamical processes. Foundations and applications in nuclear, atomic and molecular physics, Lecture Notes in Physics, Vol. 325, Springer-Verlag, Berlin, 1989.

[4] Rosas-Ortiz O., Fernández-García N., Cruz y Cruz S., A primer on resonances in quantum mechanics, AIP Conf. Proc. 1077 (2008), 31-57, arXiv:0902.4061.

[5] Rotter I., A non-Hermitian Hamilton operator and the physics of open quantum systems, J. Phys. A: Math. Theor. 42 (2009), 153001, 51 pages.

[6] García-Calderón G., Theory of resonant states: an exact analytical approach for open quantum systems, Adv. Quantum Chem. 60 (2010), 407-456.

[7] Fernández-García N., Rosas-Ortiz O., Extended WKB method, resonances and supersymmetric radial barriers, Int. J. Theor. Phys., to appear, arXiv:1103.3692.

[8] Espinosa M.G., Kielanowski P., Unstable quantum oscillator, J. Phys. Conf. Ser. 128 (2008), 012037, 7 pages.

[9] Ludviksson A., A simple model of a decaying quantum mechanical state, J. Phys. A: Math. Gen. 20 (1987), 4733-4738.

[10] Emmanouilidou A., Moiseyev N., Stark and field-born resonances of an open square well in a static external electric field, J. Chem. Phys. 122 (2005), 194101, 9 pages.

[11] Antoniou I.E., Gadella M., Hernández E., Jauregui A., Melnikov Y., Mondragón A., Pronko G.P., Gamow vectors for barrier wells, Chaos Solitons Fractals 12 (2001), 2719-2736.

[12] de la Madrid R., Gadella M., A pedestrian introduction to Gamow vectors, Amer. J. Phys. 70 (2002), 626-638, quant-ph/0201091.

[13] de la Madrid R., The rigged Hilbert space of the algebra of the one-dimensional rectangular barrier potential, J. Phys. A: Math. Gen. 37 (2004), 8129-8157, quant-ph/0407195.

[14] Zavin R., Moiseyev N., One-dimensional symmetric rectangular well: from bound to resonance via selforthogonal virtual state, J. Phys. A: Math. Gen. 37 (2004), 4619-4628.

[15] Fernández-García N., Rosas-Ortiz O., Gamow-Siegert functions and Darboux-deformed short range potentials, Ann. Physics 323 (2008), 1397-1414, arXiv:0810.5597.

[16] Klaiman S., Moiseyev N., The absolute position of a resonance peak, J. Phys. B: At. Mol. Opt. Phys. 43 (2010), 185205, 4 pages, arXiv:1005.4756.

[17] Christiansen P.L., Arnbak H.C., Zolotaryuk A.V., Ermakov V.N., Gaididei Y.B., On the existence of resonances in the transmission probability for interactions arising from derivatives of Dirac's delta function, J. Phys. A: Math. Gen. 36 (2003), 7589-7600.

[18] Flügge S., Practical quantum mechanics, Springer-Verlag, Berlin, 1999.

[19] Fernández D., Gadella M., Nieto L.M., Supersymmetry transformations for delta potentials, SIGMA 7 (2011), 029, 14 pages, arXiv:1012.0808.

[20] Scarf F.L., New soluble energy band problem, Phys. Rev. 112 (1958), 1137-1140.

[21] Negro J., Nieto L.M., Rosas-Ortiz O., On a class of supersymmetric quantum mechanical singular potentials, in Foundations of Quantum Physics, Editors R. Blanco et al., CIEMAT/RSEF, Madrid, 2002, 259-270.

[22] Negro J., Nieto L.M., Rosas-Ortiz O., Regularized Scarf potentials: energy band structure and supersymmetry, J. Phys. A: Math. Gen. 37 (2004), 10079-10093.

[23] Mielnik B., Rosas-Ortiz O., Factorization: little or great algorithm?, J. Phys. A: Math. Gen. 37 (2004), $1007-10035$.

[24] Khare A., Sukhatme U., Periodic potentials and supersymmetry, J. Phys. A: Math. Gen. 37 (2004), 1003710056, quant-ph/0402206.

[25] Baye D., Sparenberg J.-M., Inverse scattering with supersymmetric quantum mechanics, J. Phys. A: Math. Gen. 37 (2004), 10223-10249. 
[26] Andrianov A.A., Cannata F., Nonlinear supersymmetry for spectral design in quantum mechanics, J. Phys. A: Math. Gen. 37 (2004), 10297-10321, hep-th/0407077.

[27] Fernández D.J., Supersymmetric quantum mechanics, AIP Conf. Proc. 1287 (2010), 3-36, arXiv:0910.0192.

[28] Andrianov A.A., Borisov N.V., Ioffe M.V., Factorization method and Darboux transformation for multidimensional Hamiltonians, Theoret. and Math. Phys. 61 (1984), 1078-1088.

[29] Baye D., Levai G., Sparenberg J.-M., Phase-equivalent complex potentials, Nuclear Phys. A 599 (1996), 435-456.

[30] Andrianov A.A., Ioffe M.V., Cannata F., Dedonder J.-P., SUSY quantum mechanics with complex superpotentials and real energy spectra, Internat. J. Modern Phys. A 14 (1999), 2675-2688, quant-ph/9806019.

[31] Fernández D.J., Muñoz R., Ramos A., Second order SUSY transformations with 'complex energies', Phys. Lett. A 308 (2003), 11-16, quant-ph/0212026.

[32] Rosas-Ortiz O., Muñoz R., Non-Hermitian SUSY hydrogen-like Hamiltonians with real spectra, J. Phys. A: Math. Gen. 36 (2003), 8497-8506, quant-ph/0302190.

[33] Rosas-Ortiz O., Gamow vectors and supersymmetric quantum mechanics, Rev. Mexicana Fís. 53 (2007), suppl. 2, 103-109, arXiv:0810.2283.

[34] Taylor J.R., Scattering theory. The quantum theory of nonrelativistic collisions, Dover, New York, 2006.

[35] Bohm D., Quantum theory, Prentice-Hall, Englewood Cliffs, NJ, 1951.

[36] Wigner E.P., Lower limit for the energy derivative of the scattering phase shift, Phys. Rev. 98 (1955), $145-147$.

[37] Smith F.T., Lifetime matrix in collision theory, Phys. Rev. 118 (1960), 349-356.

[38] Amrein W.O., Cibils M.B., Global and Eisenbud-Wigner time delay in scattering theory, Helv. Phys. Acta 60 (1987), 481-500.

[39] Richard S., Tiedra de Aldecoa R., Time delay is a common feature of quantum scattering theory, arXiv:1008.3433.

[40] Moshinsky M., Boundary conditions and time-dependent states, Phys. Rev. 84 (1951), 525-532.

[41] Moshinsky M., Diffraction in time, Phys. Rev. 88 (1952), 625-631.

[42] Osborn T.A., Bollé D., An extended Levinson's theorem, J. Math. Phys. 18 (1977), 432-440.

[43] Pedersen M.H., van Langen S.A., Büttiker M., Charge fluctuations in quantum point contacts and chaotic cavities in the presence of transport, Phys. Rev. B 57 (1998), 1838-1846, cond-mat/9707086.

[44] Emmanouilidou A., Reichl L.E., Scattering properties of an open quantum system, Phys. Rev. A 62 (2000), 022709, 8 pages.

[45] Amrein W.O., Jacquet Ph., Time delay for one-dimensional quantum systems with steplike potentials, Phys. Rev. A 75 (2007), 022106, 20 pages, quant-ph/0610198.

[46] Barr A.M., Reichl L.E., Quasibound states in two- and three-dimensional open quantum systems, Phys. Rev. A 81 (2010), 022707, 7 pages.

[47] Huage E.H., Støvneng J.A., Tunneling times: a critical review, Rev. Mod. Phys. 61 (1989), 917-936.

[48] de Carvalho C.A.A., Nussenzveig H.M., Time delay, Phys. Rep. 364 (2002), 83-174.

[49] Sassoli de Bianchi M., Time-delay of classical and quantum scattering process: a conceptual overview and a general definition, arXiv:1010.5329.

[50] Golub R., Felber S., Gähler R., Gutsmiedl E., A modest proposal concerning tunneling times, Phys. Lett. A 148 (1990), 27-30.

[51] Huang Y.Z., Wang C.M., Comparisons of phase times with tunnelling times based on absorption probabilities, J. Phys.: Condens. Matter 3 (1991), 5915-5919.

[52] Muga J.G., Brourard S., Sala R., Equivalence between tunnelling times based on (a) absorption probabilities, (b) the Larmor clock, and (c) scattering projectors, J. Phys.: Condens. Matter 4 (1992), L579-L584.

[53] Jaworski W., Wardlaw D.M., Time delay in tunneling: transmission and reflection time delays, Phys. Rev. A 37 (1988), 2843-2854.

[54] Negro J., Nieto L.M., Rosas-Ortiz O., Confluent hypergeometric equations and related solvable potentials in quantum mechanics, J. Math. Phys. 41 (2000), 7964-7996.

[55] Rosas-Ortiz O., Negro J. and Nieto L.M., Physical sectors of the confluent hypergeometric functions space, Rev. Mexicana Fís. 49 (2000), suppl. 1, 88-94, quant-ph/0105091.

[56] Wang Z.X., Guo D.R., Special functions, World Scientific, Singapore, 1989. 